\title{
Nasal inflammation in sleep apnoea patients using CPAP and effect of heated humidification
}

\author{
I. Koutsourelakis*, E. Vagiakis*, E. Perraki*, M. Karatza*, C. Magkou* , M. Kopaka\#, \\ C. Roussos* and S. Zakynthinos*
}

ABSTRACT: Nasal continuous positive airway pressure (CPAP) can cause undesirable nasal symptoms, such as congestion to obstructive sleep apnoea (OSA) patients, whose symptoms can be attenuated by the addition of heated humidification. However, neither the nature of nasal symptoms nor the effect of heated humidification on nasal pathophysiology and pathology are convincingly known.

20 patients with OSA on nasal CPAP who exhibited symptomatic nasal obstruction were randomised to receive either 3 weeks of CPAP treatment with heated humidification or 3 weeks of CPAP treatment with sham-heated humidification, followed by 3 weeks of the opposite treatment, respectively. Nasal symptom score, nasal resistance, nasal lavage interleukin-6, interleukin-12 and tumour necrosis factor- $\alpha$ and nasal mucosa histopathology were assessed at baseline and after each treatment arm.

Heated humidification in comparison with sham-heated humidification was associated with decrease in nasal symptomatology, resistance and lavage cytokines, and attenuation of inflammatory cell infiltration and fibrosis of the nasal mucosa.

In conclusion, nasal obstruction of OSA patients on CPAP treatment is inflammatory in origin and the addition of heated humidification decreases nasal resistance and mucosal inflammation.

KEYWORDS: Continuous positive airway pressure, heated humidification, nasal obstruction, obstructive sleep apnoea

$\mathbf{N}$ asal continuous positive airway pressure (CPAP) is considered the standard of care for treating moderate-to-severe obstructive sleep apnoea (OSA) [1]. Its use has been convincingly associated with substantial improvement in quality of life [2] and somnolence [3], along with significant benefits to cardiovascular morbidity and mortality [4, 5]. The major clinical challenge however remains improving long-term adherence to CPAP [6]. Among the side effects caused by CPAP, undesirable nasal symptoms such as congestion, dryness or rhinorrhea are particularly prevalent and can significantly compromise compliance [7]. Several mechanisms may be potentially involved in the development of nasal discomfort with CPAP but they have been described only in healthy volunteers [8-10] and in a rodent model [11]. Indeed, deliberate mouth leaks while normal subjects were using CPAP can cause high unidirectional nasal airflow with increased nasal airway resistance [8] as well as enhance nasal mucosal blood flux [9]. Similarly, ToGIAs et al. [10] documented a release of inflammatory mediators in nasal-wash fluids when compressed cold and dry air was delivered through the nose. More recently, AlmENDros et al. [11] showed that CPAP employed in rats can trigger early nasal inflammation. However, it remains unknown if the same mechanisms could be responsible for the nasal side effects of CPAP in OSA patients, who frequently have increased nasal symptoms even in the absence of CPAP use [12]. Recognition of the inflammatory nature of the nasal side effects of CPAP in OSA would be important for implementation of effective treatment. Of the variety of methods used to treat nasal symptoms during CPAP treatment, the most common is heated humidification of the inspired air [13]. Although several authors have studied the effect of different forms of humidification on the relative humidity of CPAP airflow, patient adherence and subjective outcomes [13-15], no study has so far examined the functional pathways by which heated humidified CPAP attenuates nasal symptoms in OSA patients [16].
AFFILIATIONS

${ }^{*}$ Center of Sleep Disorders, Medical School of Athens University, Dept of Critical Care and Pulmonary Services, Evangelismos Hospital, and "Dept of Pathology, Evangelismos Hospital, Athens, Greece.

CORRESPONDENCE

I. Koutsourelakis

Medical School of Athens University, Center of Sleep Disorders

Evangelismos Hospital

45-47 Ipsilandou Str

GR 10675

Athens

Greece

E-mail: ykoutsourelakis@yahoo.gr

Received:

March 082010

Accepted after revision:

June 172010

First published online:

July 012010 
Therefore, the purpose of this study was to examine the nature of nasal side-effects of CPAP in OSA patients and the effect of heated humidification. A randomised, crossover, shamcontrolled design was adopted, and nasal resistance, nasal wash inflammatory markers and pathology of nasal mucosa were examined in OSA patients using CPAP and exhibiting nasal symptoms. Our hypothesis was that nasal side effects of CPAP are inflammatory in origin and the addition of heated humidification attenuates nasal mucosal inflammation and decreases the elevated nasal resistance caused by the use of CPAP.

\section{METHODS}

\section{Study subjects}

Patients who met the following criteria were considered eligible for enrolment: 1) OSA with an apnoea-hypopnoea index $>15$ events $\cdot h^{-1}$ at diagnostic polysomnography; 2 ) initiation of CPAP therapy without humidification after in-laboratory polysomnography for the manual titration of CPAP; 3) symptomatic nasal obstruction attributed to, or exacerbated by, initial nasal CPAP use; 4) no medication known to influence nasal resistance (e.g., antihistamines, vasoconstrictors, topical or systemic steroids); 5) no smoking for the previous 6 months; 6 ) no upper or lower respiratory tract disease (e.g., upper respiratory tract infection, rhinitis, sinusitis, chronic obstructive pulmonary disease), including a history of nasal allergy; and 7) written informed consent from each patient. The exclusion criterion was poor compliance with CPAP therapy during the course of the study (mean CPAP daily use $<4 \mathrm{~h}$, calculated after CPAP usage data were downloaded using proprietary software (Scan software; ResMed, Sydney, Australia)). The study protocol was approved by the hospital ethics committee.

\section{Protocol}

Using a table of random numbers, subjects were randomised into two groups. The patients of the first group $\left(\mathrm{HH}_{1}\right.$; fig. $\left.1 \mathrm{a}\right)$ initially underwent 3-week nasal CPAP therapy (S7 Escape ${ }^{\mathrm{TM}}$; ResMed) with heated humidity followed by 3-week nasal CPAP therapy with sham-heated humidity. The patients of the second group $\left(\mathrm{HH}_{2}\right.$; fig. 1a) initially underwent 3-week nasal CPAP therapy with sham-heated humidity followed by 3-week nasal CPAP therapy with heated humidity. No washout period between the 3-week regimens was employed because it was considered unethical to withhold therapy. The treatment duration of 3 weeks was chosen because nasal symptoms are known to occur shortly after CPAP exposure [16]. Heated humidity was achieved by passing air from the CPAP machine through a heating unit (HumidAire $2 \mathrm{i}^{\mathrm{TM}}$; ResMed) consisting of a heated $\left(37^{\circ} \mathrm{C}\right)$ water chamber. Sham-heated humidity was achieved by turning off the heating unit, while water was not added to the chamber. Patients were wearing the same CPAP interface (nasal mask) in both arms of the study and were contacted by an experienced technician after the first, sixth, eleventh and sixteenth day of each treatment arm in order to increase compliance and to ensure that patients were actually using the treatment modality they were prescribed.

\section{Measurements}

All patients underwent three assessments: at baseline; after the first 3-week regimen; and after the second 3-week regimen, as shown in figure 1. Each assessment took place at a specific a)
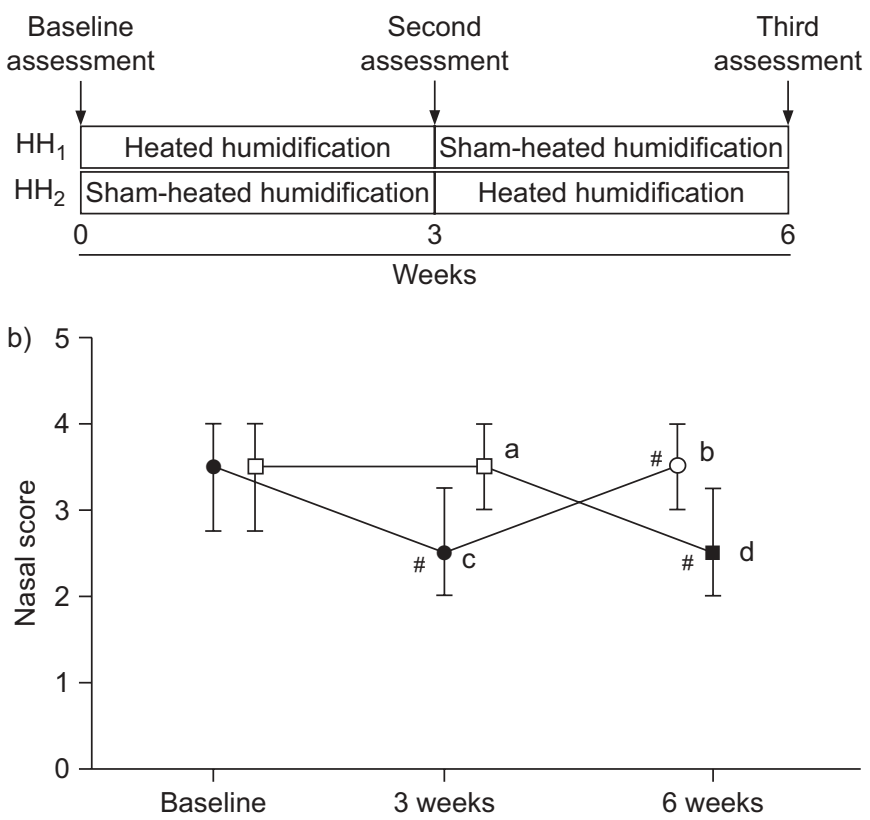

FIGURE 1. Study design and nasal score values. a) Crossover study design. After baseline assessment, the 10 patients of the first group $\left(\mathrm{HH}_{1}\right)$ underwent 3 weeks of continuous positive airway pressure (CPAP) therapy with heated humidification (-) followed by 3 weeks of CPAP therapy with sham-heated humidification $(O)$, whereas the 10 patients of the second group $\left(\mathrm{HH}_{2}\right)$ underwent 3 weeks of CPAP therapy with sham-heated humidification ( $\square$ ) followed by 3 weeks of CPAP therapy with heated humidification ( $\mathbf{\square})$. b) Nasal score decreased in both periods of administration of heated humidification, whereas sham-humidification had no effect on nasal score in $\mathrm{HH}_{2}$ and increased the already decreased by the use of heated humidification nasal score in $\mathrm{HH}_{1}$, which reached to a level comparable to that of baseline. Data are presented as median \pm interquartile range. *: $p<0.05$ versus previous assessment value. The possibility of a carryover period or other treatment effect was assessed by comparing the values of the differences at the end of each period between $\mathrm{HH}_{1}$ and $\mathrm{HH}_{2}$ groups with Mann-Whitney $\mathrm{U}$ signed rank sum test ( $d$ minus a versus $c$ minus $b$ ) with "heated humidification independent" $p$-value (phhi) indicating significance. The treatment effect of heated humidification (individual crossover difference between heated humidification and sham-heated humidification) was analysed by comparing the values at the end of each period ( $a$ plus $b$ versus $c$ plus $d$ ) with Wilcoxon-matched paired test.

time (between 10:00 $\mathrm{h}$ and 11:00 $\mathrm{h}$ ) and consisted of filling in the nasal symptoms questionnaire and performing anterior rhinomanometry in the seated and supine positions, nasal lavage and nasal mucosa biopsies.

\section{Nasal symptoms questionnaire}

Nasal symptoms were assessed using a five-point nasal scale score, as previously described [17]. Accordingly, rhinorrhea, post-nasal drip, sneezing, impaired sense of smell and nasal blockage were binary coded as present/increased over baseline (1) or absent/not increased (0) and summed to yield a total nasal score between zero and five.

\section{Rhinomanometry}

For each subject, nasal resistance to airflow was measured during wakefulness, first in the upright seated position and then in the supine position by active anterior rhinomanometry 
(PDD-301/r; Piston, Budapest, Hungary) using a standard protocol [18]. In brief, patients wore a closely fitting face mask which didn't distort the nostrils or the nasal valve and breathed through one nostril only (first the left and afterwards the right) with the mouth closed. The pressure probe was placed at the opening of the contralateral occluded nostril not being tested. Total resistance was then automatically calculated from the two unilateral measurements. Nasal resistance was given at a pressure difference of $150 \mathrm{~Pa}$ across the nasal passage. Nasal resistance values below $3.0 \mathrm{cmH}_{2} \mathrm{O} \cdot \mathrm{s} \cdot \mathrm{L}^{-1}$ were considered within normal limits [18].

\section{Nasal lavage procedure and analysis}

Nasal isotonic saline lavages were carried out using a technique previously described [17]. In brief, a 12-F Foley catheter (Bard, Crawley, UK), modified by the removal of the tip distal to the balloon, was inserted into the nostril and inflated with sufficient air to form a comfortable seal (typically $7-10 \mathrm{~mL}$ ). With the patients head flexed $45^{\circ}$ forward, $7 \mathrm{~mL}$ of warmed $0.9 \%$ saline was instilled through the catheter and washed in and out of the nasal cavity three times. The lavage fluids from both nostrils were centrifuged $(325 \times \mathrm{g}$, $10 \mathrm{~min}$, $4{ }^{\circ} \mathrm{C}$ ) and samples were obtained from the supernatant and frozen $\left(-80^{\circ} \mathrm{C}\right)$ awaiting subsequent analysis of inflammatory mediators. The inflammatory mediators interleukin (IL)-6, IL-12 and tumour necrosis factor (TNF)- $\alpha$ were quantified using commercial sandwich high sensitivity ELISA kits (R\&D systems, Inc., Minneapolis, MN, USA). The limits of detection were $0.70,5.0$ and $1.6 \mathrm{pg} \cdot \mathrm{mL}^{-1}$ for IL-6, IL-12 and TNF- $\alpha$, respectively.

\section{Nasal biopsies}

Paired nasal biopsies were obtained. Topical anaesthesia was applied using a solution of lidocaine and naphazoline delivered first by a spray device and thereafter by a cotton swab. Nasal biopsies were extracted from the inferior aspects of the inferior nasal turbinate $\sim 0.5 \mathrm{~cm}$ from its anterior margin, taking care not to crush the tissue. A cutting punch forcep (diameter $2 \mathrm{~mm}$ ) with a drilled-out punch was used for this purpose. The specimens were immersed in $4 \%$ buffered formaldehyde, dehydrated and embedded in paraffin. Sections cut at $4 \mu \mathrm{m}$ were stained with haematoxylin-eosin and periodic-acid Schiff and analysed in a blinded fashion by two independent pathologists (C. Magkou and M. Kopaka) with a Zeis Axiophot microscope (Zeiss, Oberkochen, Germany). Semiquantitive grading was performed for the degree of inflammatory cell infiltration and fibrosis and the density of submucosal mucous glands according to the criteria of MAURIELLO et al. [19]. In brief, the degree of inflammatory cell infiltration or fibrosis and the density of submucosal mucous glands were considered: 1) absent, when they could not be detected in the specimen; 2) mild and moderate, when they were confined to the upper one-third and one-half of the subepithelial mucosa, respectively; and 3) severe, when they were extended beyond the upper half or involved the full thickness of the subepithelial mucosa [19].

\section{Statistical analysis}

Statistical procedures were based on recommendations for analysis of crossover trials [20] and included analyses of heatedhumidification dependent and sham-heated-humidification dependent effects (see fig. 1). Non-parametric statistics were applied. Differences between group characteristics at baseline in quantitative and qualitative data were evaluated by the Mann-Whitney U-test and Fisher's exact test, respectively. Within-group changes of variables were analysed with Wilcoxon-matched paired test, when indicated. The treatment effect of heated humidification (individual crossover difference between heated humidification and sham-heated humidification) was analysed by comparing the values at the end of each period with Wilcoxon-matched paired test with "heatedhumidification dependent" $p$-value (phhd) indicating significance. The possibility of a carryover period or other treatment effect was assessed by comparing the values of the differences at the end of each period between $\mathrm{HH}_{1}$ and $\mathrm{HH}_{2}$ groups with Mann-Whitney U signed rank sum test with "heatedhumidification independent" p-value (phi) indicating significance (fig. 1). The Bonferroni correction was used for multiple comparisons. Reported p-values are two-sided and pertain to the variables that remained statistically significant after the Bonferroni correction. An a priori power analysis was performed with GPower 3.1 (Franz Faul, University of Kiel, Germany). The minimum sample size was calculated based on $90 \%$ power and a two-sided 0.05 significance level. The sample size capable of detecting a between-condition difference of $0.3 \mathrm{cmH}_{2} \mathrm{O} \cdot \mathrm{s} \cdot \mathrm{L}^{-1}$ was estimated for the decrease in nasal resistance using data from previous studies $[8,21]$. The critical sample size was estimated to be 20 patients. Statistical significance was accepted at $\mathrm{p}<0.05$. Data are presented as median (interquartile range).

\section{RESULTS}

Of the 28 patients (21 males) consecutively examined for eligibility, 20 patients were enrolled in the study, and eight patients were not included (three patients with respiratory tract infections, three patients with a history of nasal allergy or sinusitis and topical steroid use, and two patients with chronic obstructive pulmonary disease). 10 patients were randomly assigned to the $\mathrm{HH}_{1}$ group and 10 patients to the $\mathrm{HH}_{2}$ group; all of them finished the study uneventfully.

\section{Baseline characteristics}

No significant differences were evident between the two groups (table 1).

\section{Nasal score}

Nasal score decreased after using heated humidification in comparison with sham-heated humidification (2.30 (2.0-3.00) versus 3.40 (3.00-4.00), phhd $<0.001)$. Nasal score decreased in both periods of administration of heated humidification, whereas sham-humidification had no effect on nasal score in $\mathrm{HH}_{2}$ and increased the already decreased by the use of heated humidification nasal score in $\mathrm{HH}_{1}$, which reached to a level comparable to that of baseline (fig. 1b).

\section{Nasal resistance}

Nasal resistance in supine position decreased after administration of heated humidification in comparison with shamheated humidification (2.3 (2.00-2.50) versus 2.8 (2.4-3.5) $\mathrm{cmH}_{2} \mathrm{O} \cdot \mathrm{s} \cdot \mathrm{L}^{-1}$, phhd $\left.<0.001\right)$. Nasal resistance decreased in both periods of applying heated humidification, whereas shamhumidification had no effect on nasal resistance in $\mathrm{HH}_{2}$ and 


\begin{tabular}{|c|c|c|}
\hline & $\mathrm{HH}_{1}$ group & $\mathrm{HH}_{2}$ group \\
\hline Subjects n & 10 & 10 \\
\hline Age yrs & $61.5(51.0-67.0)$ & $62.0(48.8-68.5)$ \\
\hline Male sex $n$ & 6 & 6 \\
\hline Body mass index $\mathrm{kg} \cdot \mathrm{m}^{-2}$ & $31.5(28.8-35.3)$ & $30.5(28.8-33.3)$ \\
\hline $\begin{array}{l}\text { Apnoea-hypopnoea index } \\
\text { events } \cdot h^{-1}\end{array}$ & $32.4(26.8-38.3)$ & $34.0(28.7-39.5)$ \\
\hline $\begin{array}{l}\text { Nasal resistance supine } \\
\mathrm{cmH}_{2} \mathrm{O} \cdot \mathbf{s} \cdot \mathrm{L}^{-1}\end{array}$ & $2.7(2.4-3.2)$ & $2.7(2.3-3.3)$ \\
\hline $\begin{array}{l}\text { Nasal resistance seated } \\
\mathrm{cmH}_{2} \mathrm{O} \cdot \mathbf{s} \cdot \mathrm{L}^{-1}\end{array}$ & $2.6(2.3-3.2)$ & $2.5(2.3-3.1)$ \\
\hline Nasal score & $3.5(2.8-4.0)$ & $3.5(2.8-4.0)$ \\
\hline CPAP pressure $\mathrm{cmH}_{2} \mathrm{O}$ & $7.5(6.3-8.5)$ & $7.2(6.1-8.2)$ \\
\hline CPAP use at enrolment days & $11(5-20)$ & $9(5-17)$ \\
\hline \multicolumn{3}{|c|}{$\begin{array}{l}\text { Data are presented as median (interquartile range), unless otherwise stated. } \\
\mathrm{HH}_{1} \text { group: patients initially underwent } 3 \text { weeks of continuous positive airway } \\
\text { pressure (CPAP) therapy with heated humidification followed by } 3 \text { weeks of } \\
\mathrm{CPAP} \text { therapy with sham-heated humidification; } \mathrm{HH}_{2} \text { group: patients initially } \\
\text { underwent } 3 \text { weeks of CPAP therapy with sham-heated humidification followed } \\
\text { by } 3 \text { weeks of CPAP therapy with heated humidification; nasal score: a five- } \\
\text { point nasal symptom scale score based on a nasal symptoms questionnaire } \\
\text { yielding a score between zero and five [17]. }\end{array}$} \\
\hline
\end{tabular}

increased the previously decreased by the application of heated humidification nasal resistance in $\mathrm{HH}_{1}$, which reversed to levels similar to that of baseline (fig. 2). Results of nasal resistance in seated position were identical.

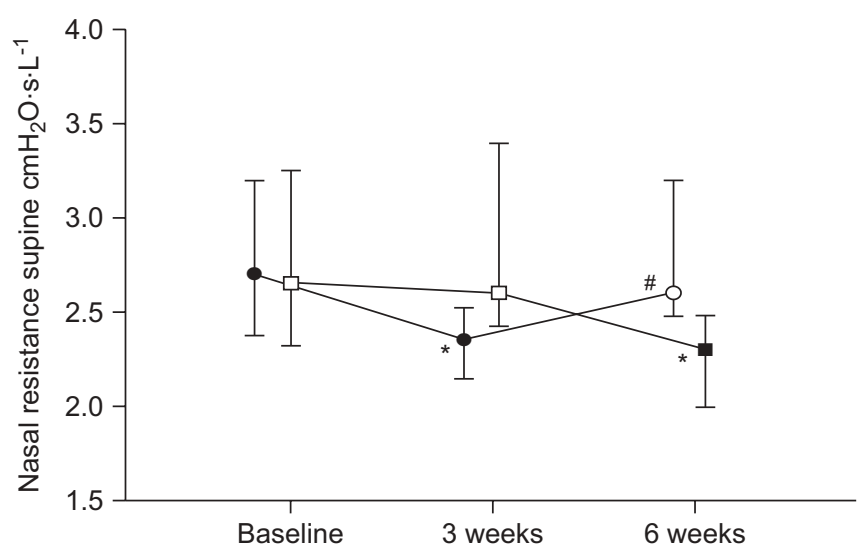

FIGURE 2. Nasal resistance values. Nasal resistance decreased in both periods of applying heated humidification, whereas sham-humidification had no effect on nasal resistance in $\mathrm{HH}_{2}$ group and increased the previously decreased by the application of heated humidification nasal resistance in $\mathrm{HH}_{1}$ group, which reversed to levels similar to that of baseline. $-\mathrm{HH}_{1}$ group heated humidification; $\mathrm{O}: \mathrm{HH}_{1}$ group sham heated humidification; $\mathbf{a}: \mathrm{HH}_{2}$ group heated humidification; $\square: \mathrm{HH}_{2}$ group sham heated humidification. *: $\mathrm{p}<0.01$ versus previous assessment value; ${ }^{\#}: p<0.05$ versus previous assessment value. For further explanation see legend of fig. 1.

\section{Nasal lavage inflammatory mediators}

Baseline nasal lavage levels of IL-6, IL-12 and TNF- $\alpha$ were not different between $\mathrm{HH}_{1}$ and $\mathrm{HH}_{2}$ groups, and levels of these cytokines decreased after administration of heated humidification in comparison with sham-heated humidification (1.2 (1.01.5) versus $1.5(1.3-1.6) \mathrm{pg} \cdot \mathrm{mL}^{-1}$ for IL-6, phhd $<0.001 ; 7.2$ (6.2-7.9) versus 8.3 (7.4-9.1) $\mathrm{pg} \cdot \mathrm{mL}^{-1}$ for IL-12, phhd $=0.005$; and $1.8(1.7-2.1)$ versus $2.6(1.9-2.7) \mathrm{pg} \cdot \mathrm{mL}^{-1}$ for TNF- $\alpha$, phhd $\left.=0.001\right)$. All cytokine levels decreased in both periods of using heated humidification, whereas sham-humidification had no effect on cytokine levels in $\mathrm{HH}_{2}$ and increased the previously decreased by the application of heated humidification cytokine levels in $\mathrm{HH}_{1}$, which reversed to levels similar to that at baseline (fig. 3).

\section{Nasal mucosa pathology}

Nasal mucosa specimens revealed a chronic subepithelial inflammatory cell infiltration consisting predominantly of lymphocytes and plasma cells. At baseline, the degree of inflammation and fibrosis and the density of the submucosal mucous glands were almost identical between $\mathrm{HH}_{1}$ and $\mathrm{HH}_{2}$ groups (data not shown). The administration of heated humidification was associated with a significant decrease in the degree of inflammation and fibrosis in comparison with sham-heated humidification (table 2). Representative specimens are shown in fig. 4.

\section{Carryover effect}

Carryover or treatment effect of the application of heated humidification on the result of sham-heated humidification in $\mathrm{HH}_{1}$ group and of the application of sham-heated humidification on the result of heated humidification in $\mathrm{HH}_{2}$ group was not significant for nasal score, nasal resistance, and nasal lavage cytokines (figs 1b, 2 and 3, respectively). Therefore, it can be assumed that possible carryover period or treatment effect could not have influenced the results.

\section{DISCUSSION}

The data of this study have demonstrated the following: 1) the inflammatory nature of nasal side effects of CPAP in OSA patients; and 2) that a 3-week administration of CPAP with heated humidification in comparison with sham-heated humidification was associated with decrease in nasal symptomatology, resistance and lavage cytokines (IL-6, IL-12, TNF- $\alpha$ ), and attenuation of inflammatory and fibrotic changes of the nasal mucosa.

Nasal discomfort is among the most common side effects that CPAP can cause in OSA patients and nasal side effects can compromise long-term compliance to CPAP therapy [6]. Previous studies have assessed the role of CPAP on nasal airway of healthy volunteers [8-10, 16] and in a rodent model [11], and have also evaluated the effect of different forms of humidification on nasal symptoms and adherence in OSA patients [13-15, 22]. However, the effect of CPAP (with and without humidification) on nasal airway pathophysiology of OSA patients remains unknown. The current study adds to the literature by demonstrating that CPAP with heated humidification reduces preexisting nasal airway inflammation and nasal symptomatology, presumably attributed to previous use of CPAP without heated humidification. OSA patients on CPAP therapy exhibiting nasal obstruction were recruited and nasal airway pathophysiology and pathology were evaluated. This subgroup of OSA patients 

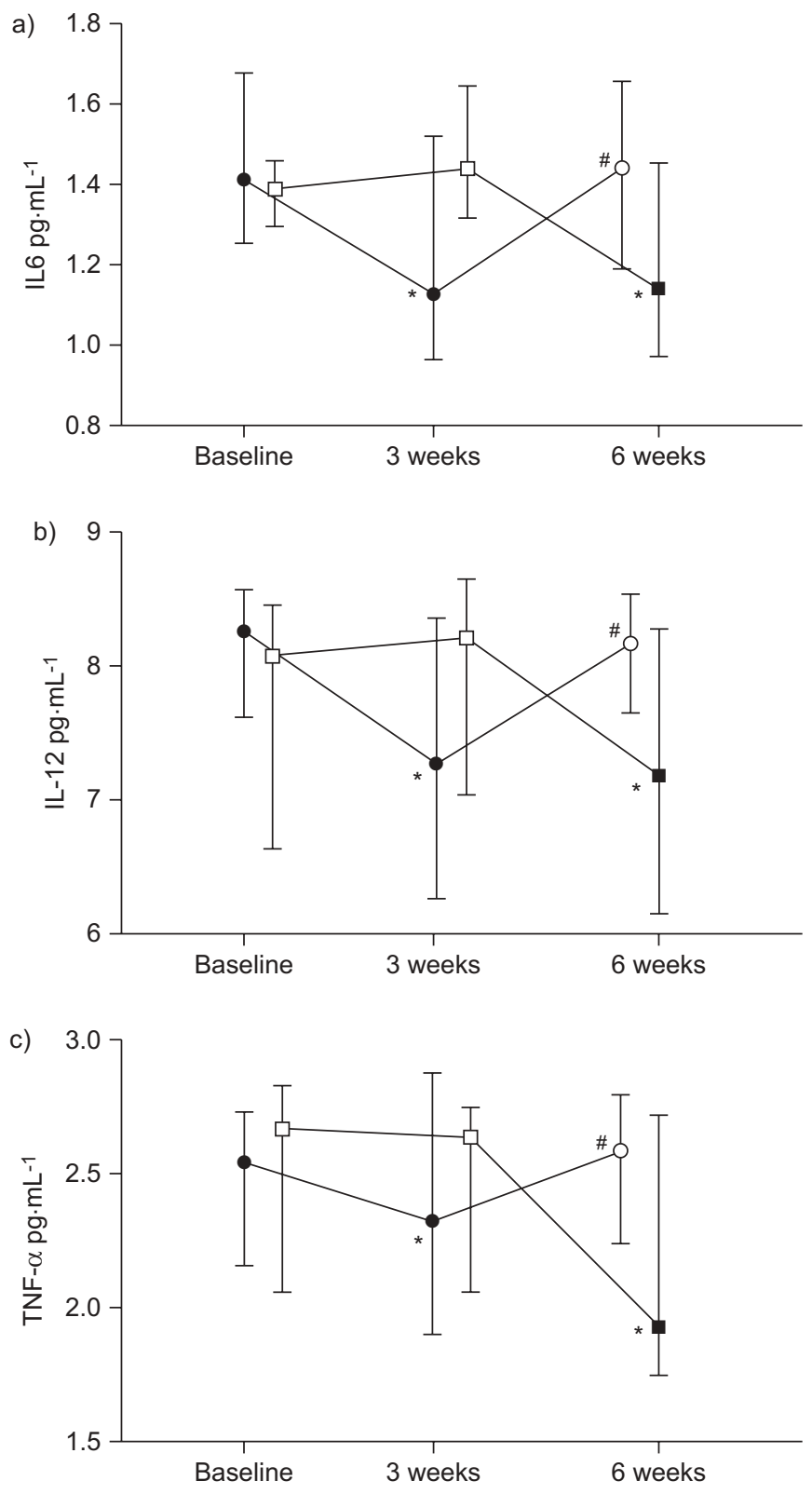

FIGURE 3. a) Interleukin (IL)-6, b) IL-12, and c) tumour necrosis factor (TNF)- $\alpha$ values in nasal lavage fluids. All cytokine levels decreased in both periods of using heated humidification, whereas sham-humidification had no effect on cytokine levels in $\mathrm{HH}_{2}$ group and increased the previously decreased by the application of heated humidification cytokine levels in $\mathrm{HH}_{1}$ group, which reversed to levels similar to that at baseline. $-\mathrm{HH}_{1}$ group heated humidification; $\mathrm{O}: \mathrm{HH}_{1}$ group sham heated humidification; $\mathbf{\square}: \mathrm{HH}_{2}$ group heated humidification; $\square: \mathrm{HH}_{2}$ group sham heated humidification. *: $p<0.01$ versus previous assessment value; ${ }^{*}: p<0.05$ versus previous assessment value. For further explanation see legend of fig. 1 .

was selected because it is in these patients that the application of $\mathrm{CPAP}$ had already provoked clinical problems and thus the effect of continuation of CPAP either with sham-heated humidification or heated humidification on nasal airway would be more apparent and clinically relevant.

Only two previous studies that included a small number of patients have provided limited, albeit conflicting, data regarding

\begin{tabular}{|c|c|c|c|c|c|c|}
\hline \multirow[t]{3}{*}{ TABLE 2} & \multicolumn{6}{|c|}{$\begin{array}{l}\text { Histopathologic features of nasal mucosa biopsy } \\
\text { specimens after sham-heated humidification and } \\
\text { heated humidification }\end{array}$} \\
\hline & \multicolumn{2}{|c|}{ Inflammation } & \multicolumn{2}{|c|}{ Fibrosis } & \multicolumn{2}{|c|}{ Mucous glands } \\
\hline & S-HH & $\mathrm{HH}$ & S-HH & $\mathrm{HH}$ & S-HH & $\mathrm{HH}$ \\
\hline Absent & $0(0)$ & $8(40)^{\star \star}$ & $0(0)$ & $6(30)^{*}$ & $0(0)$ & $4(20)$ \\
\hline Mild & $6(30)$ & $8(40)$ & $10(50)$ & $6(30)$ & $8(40)$ & $10(50)$ \\
\hline Moderate & $8(40)$ & $4(20)$ & $6(30)$ & $8(40)$ & $8(40)$ & $6(30)$ \\
\hline Severe & $6(30)$ & $0(0)^{*}$ & 4 (20) & $0(0)$ & $4(20)$ & $0(0)$ \\
\hline $\begin{array}{l}\text { Data are pres } \\
\text { heated humid } \\
\mathrm{HH} \text {. }\end{array}$ & ation $(n=$ & $\begin{array}{l}\text { S-HH: } \mathrm{s} \\
*: \mathrm{p}<0\end{array}$ & $\begin{array}{l}\text { am-heat } \\
5 \text { versus }\end{array}$ & $\begin{array}{l}\text { A humidif } \\
\mathrm{S}-\mathrm{HH} ;\end{array}$ & $\begin{array}{l}\text { ation }(n= \\
p<0.01\end{array}$ & $\begin{array}{l}\text { 20); HH: } \\
\text { ersus S- }\end{array}$ \\
\hline
\end{tabular}

the effect of CPAP therapy on nasal pathophysiology of OSA patients [21, 23]. Indeed, CONSTANTINIDIS et al. [23] demonstrated prolonged mucociliary transport time after 3-10 months of CPAP use along with a desiccation of the epithelial surface and an inflammatory cell infiltration of the mucosa, whereas Bossi et al. [21] failed to discern any change in mucociliary transport time or in nasal resistance after 6 months of CPAP use. However, although measures of mucociliary clearance are simple to perform, they have poor reproducibility and consequently their incorporation into clinical trials is considered questionable [24].

Nasal symptomatology during CPAP therapy has been studied in OSA patients in crossover studies, a marked decrease was demonstrated in upper airway symptoms after the application of CPAP with heated humidification in comparison with CPAP with placebo humidification [14, 22]. Interestingly, ALMENDROS et al. [11], in an effort to explain nasal symptoms following $\mathrm{CPAP}$, proposed the presence of deficiency of adaptive antiinflammatory mechanisms because of pre-existing conditions such as nasal obstruction. In such a case, the application of CPAP would act as a second-hit source of injury [11]. The results obtained in the current study suggest that the addition of heated humidification could compensate for the potential deficiency associated with nasal obstruction and could thus ameliorate nasal symptomatology.

Heated humidification was associated with a decrease in the level of nasal lavage pro-inflammatory cytokines. IL-6 and TNF- $\alpha$ are important in the induction of the acute phase response of inflammation, whereas IL-12 mediates an enhancement of the cytotoxic activity of cytotoxic T-lymphocytes [25]. Although the pathophysiologic mechanisms by which CPAP causes cytokine release are unknown, some speculations can be made. It is likely that airflow of cold and dry air has a toxic effect on nasal epithelium [26] and mediator containing cells of the nasal mucosa [10]. The addition of heated humidification could eventually prevent this effect, thus avoiding epithelial detachment and shedding and mediator release. Furthermore, CPAP airflow may lead to water evaporation and an increase in the osmolarity of the extracellular fluid surrounding the mast cells that is sufficient to induce mediator release [27]. This effect could also eventually be avoided by heated 

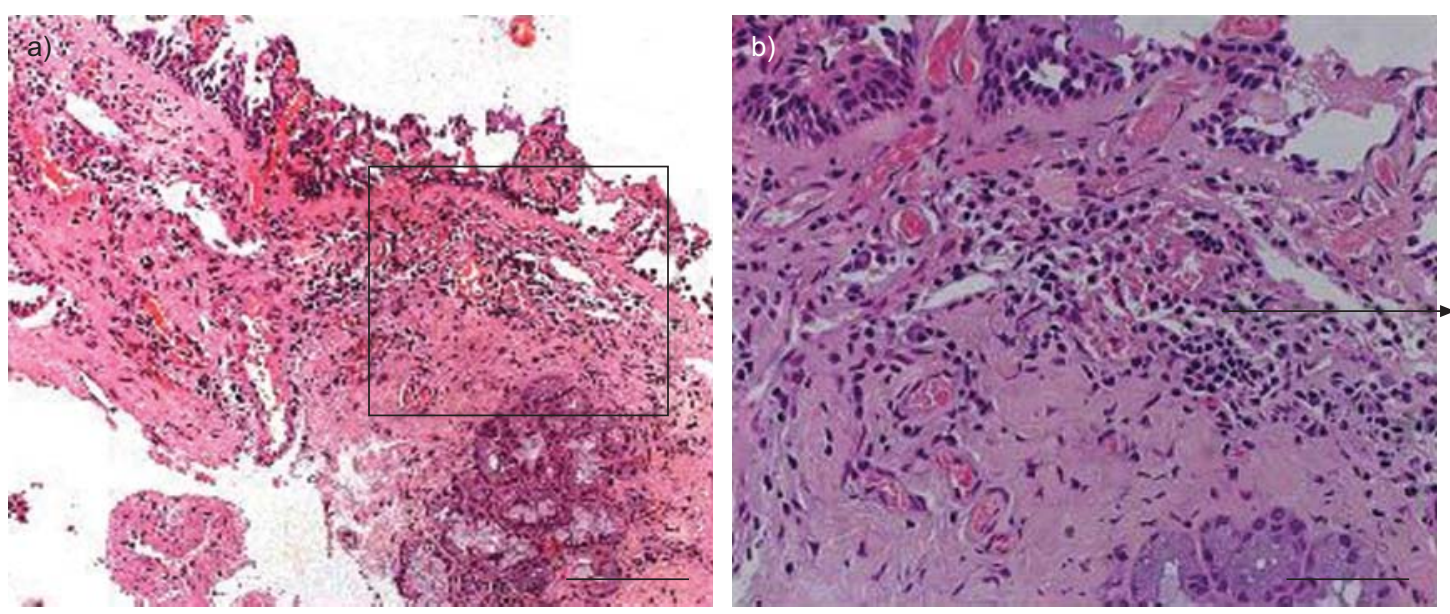

Plasma cells and lymphocytes.

Severe inflammatory

inflitration
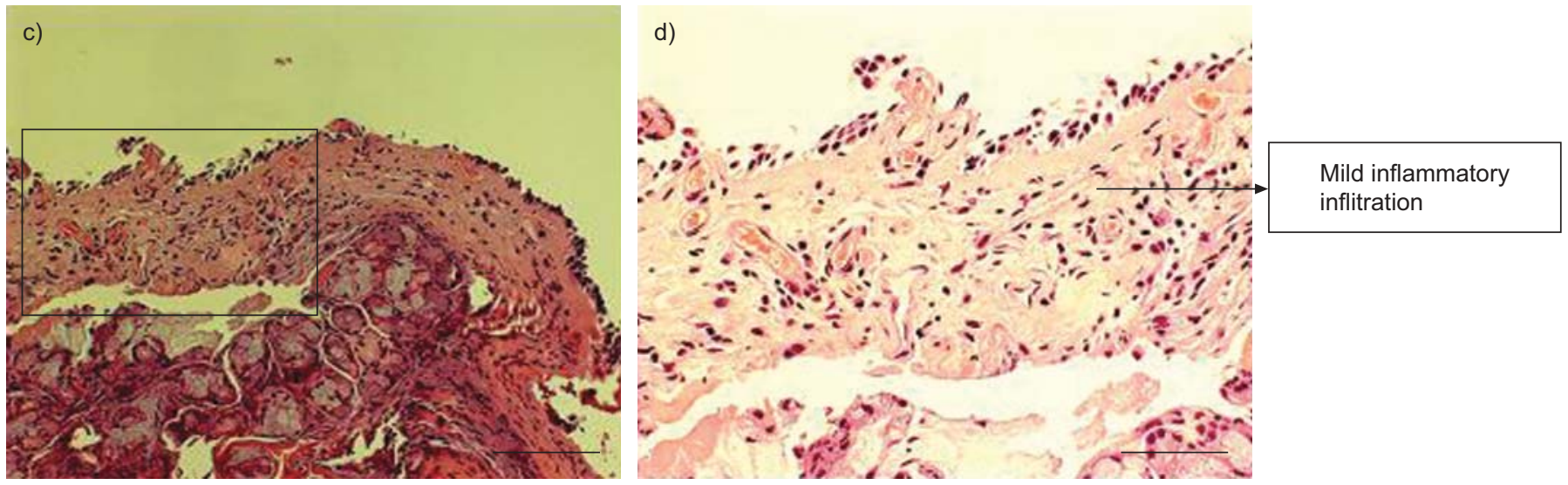

FIGURE 4. Histopathologic features of nasal mucosa biopsy specimens obtained after the administration of 3 weeks of CPAP therapy with sham-heated humidification at a) low magnification (haematoxylin-eosin stain) and b) high magnification of the black boxed area in a) (original magnification $\times 400$ ). Specimen was characterised by epithelial detachment and severe inflammatory cell infiltration; the administration of 3 weeks of CPAP therapy with heated humidification, at c) low magnification (haematoxylin-eosin) and d) high magnification of the black boxed area in c) (original magnification $\times 400$ ). Specimen shows mild inflammatory cell infiltration. a and c) Scale bars $=60 \mu \mathrm{m} ; \mathrm{b}$ and $d)$ scale bars $=30 \mu \mathrm{m}$.

humidification, as ARAújo et al. [28] have demonstrated a decreased dehydration effect of the inspired air when humidification was added to CPAP therapy.

As far as the histopathology of the nasal mucosa is concerned, the present study demonstrated an attenuated nasal mucosa inflammation after the addition of heated humidification in comparison with sham-heated humidification. This finding supports the results of a previous study, which provided evidence that CPAP can disrupt the equilibrium of the mucous membrane, giving rise to shedding and inflammation of the nasal mucosa [23]. Interestingly and in accordance with our results, CRUZ et al. [26] demonstrated that it was cold and dry air and not warm and moist air that can lead to epithelial cell damage suggesting that this effect does not result from the sheer force stimulus exerted by the airflow. They further hypothesised that the epithelial detachment following cold and dry air is likely to be linked to some form of a mucosal water transportation defect [26]. This suggestion could also provide an explanation for the attenuation of nasal inflammation after the application of heated humidification observed in our study.

Combining the results of nasal mucosa pathology with the other measurements, it is noteworthy to add that the apparition of increased nasal mucosa inflammation after application of CPAP with sham-heated humidification in $\mathrm{HH}_{1}$ group was associated with increased nasal scale score, elevated nasal resistance and increased nasal lavage inflammatory mediators (figs $1 \mathrm{~b}, 2$ and 3 , respectively). In contrast, the vanishing of nasal mucosa inflammation after application of CPAP with heated humidification in $\mathrm{HH}_{1}$ and $\mathrm{HH}_{2}$ groups was associated with a decreased nasal scale score, nasal resistance and nasal lavage inflammatory mediators (figs $1 \mathrm{~b}, 2$ and 3, respectively). Therefore, it is plausible to suggest that the time response of nasal mucosa pathology to the application of CPAP is closely reflected by the fluctuations of nasal score, nasal resistance and nasal lavage mediators.

Some methodological issues require consideration in the current study. First, blinding of the patients on the therapy used could not have been optimal. Therefore, in an attempt to control for a placebo effect, all study patients were naïve to the use of heated humidification while told that the purpose of the study was to compare the effect of CPAP treatment at "two levels of humidity". However, the objective assessment of CPAP use by the CPAP data card did not differ between arms, supporting the view that patients believed they were in fact getting a similar amount of treatment. Secondly, recommendations for nasal wash 
technique are not currently available. However, the reproducibility of the nasal wash technique that was employed in the present study for the assessment of inflammatory cytokines has been tested previously [17]. Additionally, the nasal symptom questionnaire that was used in the current study, albeit not validated, is considered a practical, rapid and useful indication of upper airway involvement and has been shown to correlate well with the more complex sino-nasal outcome test-20 (SNOT-20) questionnaire [17]. The SNOT-20 is one of the most widely used quality-of-life instruments for a validated evaluation of sino-nasal conditions and is intended for populations with rhinosinusitis [17]. Lastly, the sample size of the current study could be considered limited, especially in comparison with recently published data [29]. Yet, in accordance with the results of the present study, RYAN et al. [29] demonstrated that the addition of heated humidification is associated with a decrease in the frequency of nasal symptoms in OSA patients. However, this decrease does not lead to improved adherence to CPAP [30].

In conclusion, the present study provided evidence about the inflammatory effect of CPAP on nasal mucosa of OSA patients and that CPAP with heated humidification is associated with decrease in nasal symptomatology, resistance and lavage cytokines, and attenuation of inflammation and fibrosis of the nasal mucosa. It is thus plausible to suggest that clinical response to anti-inflammatory drugs might be anticipated. Further studies are warranted in this direction.

\section{SUPPORT STATEMENT}

The present work was funded by the Thorax Foundation and the Greek State Scholarships Foundation.

\section{CLINICAL TRIAL}

Trial Registration clinicaltrials.gov Identifier: NCT00850876.

\section{STATEMENT OF INTEREST}

None declared.

\section{REFERENCES}

1 Kushida C, Littner M, Hirshkowitz M, et al. Practice parameters for the use of continuous and bilevel positive airway pressure devices to treat adult patients with sleep-related breathing disorders. Sleep 2006; 29: 375-380.

2 Ballester E, Badia JR, Hernadez L, et al. Evidence of the effectiveness of continuous positive airway pressure in the treatment of sleep apnea/hypopnea syndrome. Am J Respir Crit Care Med 1999; 159: 495-501.

3 Patel S, White D, Malhotra A, et al. Continuous positive airway pressure therapy for treating sleepiness in a diverse population with obstructive sleep apnea. Results of a meta-analysis. Arch Intern Med 2003; 163: 565-571.

4 Marin JM, Carrizo SJ, Vicente E, et al. Long-term cardiovascular outcomes in men with obstructive sleep apnoea-hypopnoea with or without treatment with continuous positive airway pressure: an observational study. Lancet 2005; 365: 1046-1053.

5 Basner R. Continuous positive airway pressure for obstructive sleep apnea. N Engl J Med 2007; 356: 1751-1758.

6 Budhiraja R, Parthasarathy S, Drake C, et al. Early CPAP use identifies subsequent adherence to CPAP therapy. Sleep 2007; 30: 320-324.

7 Pepin JL, Leger P, Veale D, et al. Side effects of nasal continuous positive airway pressure in sleep apnea syndrome. Study of 193 patients in two French sleep centers. Chest 1995; 107: 375-381.
8 Richards G, Cistulli P, Ungar R, et al. Mouth leak with nasal continuous positive airway pressure increases nasal airway resistance. Am J Respir Crit Care Med 1996; 154: 182-186.

9 Hayes MJ, McGregor FB, Roberts DN, et al. Continuous nasal positive airway pressure with a mouth leak: effect on nasal mucosal blood flux and nasal geometry. Thorax 1995; 50: 1179-1182.

10 Togias A, Naclerio R, Proud D, et al. Nasal challenge with cold, dry air results in release of inflammatory mediators: possible mast cell involvement. J Clin Invest 1985; 76: 1375-1381.

11 Almendros I, Acerbi I, Vilaseca I, et al. Continuous positive airway pressure (CPAP) induces early nasal inflammation. Sleep 2008; 31: 127-131.

12 Lofaso F, Cote A, d'Ortho MP, et al. Nasal obstruction as a risk factor for sleep apnoea syndrome. Eur Respir J 2000; 16: 639-643.

13 Randerath W, Meier J, Genger H, et al. Effect of cold passover and heated humidification under continuous positive airway pressure. Eur Respir J 2002; 20: 183-186.

14 Massie CA, Hart RW, Peralez K, et al. Effects of humidification on nasal symptoms and compliance in sleep apnea patients using continuous positive airway pressure. Chest 1999; 116: 403-408.

15 Mador MJ, Krauza M, Pervez A, et al. Effect of heated humidification on compliance and quality of life in patients with sleep apnea using nasal continuous positive airway pressure. Chest 2005; 128: 2151-2158.

16 Willing S, San Pedro M, Driver HS, et al. The acute impact of continuous positive airway pressure on nasal resistance: a randomized controlled comparison. J Appl Physiol 2007; 102: 1214-1219.

17 Hurst J, Perera W, Wilkinson T, et al. Systemic and upper and lower airway inflammation at exacerbation of chronic obstructive pulmonary disease. Am J Respir Crit Care Med 2006; 173: 71-78.

18 Koutsourelakis I, Vagiakis E, Roussos C, et al. Obstructive sleep apnoea and oral breathing in patients free of nasal obstruction. Eur Respir J 2006; 28: 1222-1228.

19 Mauriello JA, Palydowycz S, DeLuca J. Clinicopathologic study of lacrimal sac and nasal mucosa in 44 patients with complete acquired nasolacrimal duct obstruction. Ophthal Plast Reconstr Surg 1992; 8: 13-21.

20 Senn S, ed. Cross-over Trials in Clinical Research, 1st Edn. New York, John Wiley \& Sons, 1993.

21 Bossi R, Piatti G, Roma E, et al. Effects of long-term nasal continuous positive airway pressure therapy on morphology, function, and mucociliary clearance of nasal epithelium in patients with obstructive sleep apnea syndrome. Laryngoscope 2004; 114: 1431-1434.

22 Neill AM, Wai HS, Bannan SP, et al. Humidified nasal continuous positive airway pressure in obstructive sleep apnoea. Eur Respir J 2003; 22: 258-262.

23 Constantinidis J, Knøbber D, Steinhart H, et al. Fine-structural investigations of the effect of nCPAP-mask application on the nasal mucosa. Acta Otolaryngol 2000; 120: 432-437.

24 Nathan RA, Eccles R, Howarth PH, et al. Objective monitoring of nasal patency and nasal physiology in rhinitis. J Allergy Clin Immunol 2005; 115: S442-S459.

25 Roponen M, Seuri M, Nevalainen A, et al. Nasal lavage method in the monitoring of upper airway inflammation: seasonal and individual variation. Inhal Toxicol 2003; 15: 649-661.

26 Cruz AA, Naclerio R, Proud D, et al. Epithelial shedding is associated with nasal reactions to cold, dry air. J Allergy Clin Immunol 2006; 117: 1351-1356.

27 Findlay S, Dvorak A, Kagey-Sobotka A, et al. Hyperosmolar triggering of histamine release from human baseophils. J Clin Invest 1981; 67: 1604-1613.

28 Araújo MD MT, Vieira SB, Vasquez EC, et al. Heated humidification or face mask to prevent upper airway dryness during 
continuous positive airway pressure therapy. Chest 2000; 117 : 142-147.

29 Ryan S, Doherty LS, Nolan GM, et al. Effects of heated humidification and topical steroids on compliance, nasal symptoms, and quality of life in patients with obstructive sleep apnea syndrome using nasal continuous positive airway pressure. J Clin Sleep Med 2009; 5: 422-427.

30 Worsnop CJ, Miseski S, Rochford PD. Routine use of humidification with nasal continuous positive airway pressure. Intern Med J 2010; 40: 650-656. 